\title{
Calculation and Measurement of Two-Dimensional Electromagnetic Field Distribution in Planar Circuit
}

\author{
H. Jui-Pang and T. Anada \\ Faculty of Engineering, Kanagawa University \\ 3-27-1 Rokkakubashi Kanagawaku, Yokohama-shi 221, JAPAN
}

\begin{abstract}
A detailed investigation of the two dimensional electromagnetic field distribution in the pla. nar circuit is important and useful not only for the optimized design and adjustment of planar circuits, but also the theoretical analysis of more complicated discontinuity problems. In this paper, we are described how to calculate and measure the two dimensional electromagnetic field distribution in planar circuits, making use of a kind of analogy existing between $H$-plane short-boundary planar circuit and Trough-type surface-wave planar circuit. The measured results are in good agreement with the theoretical results predicted by a numerical analysis.
\end{abstract}

\section{I.Introduction}

With the development of various MIC's, it becomes increasingly important to proceed a rigorous full electromagnetic analysis in the planar circuits.

It is considered that a detailed investigation of two dimensional electromagnetic(EM) field distribution, in addition to input/output frequency characteristics of planar circuits, is useful not only for the design and adjustment of the planar circuit, but also the prediction with the theoretical analysis of more complicated discontinuity problems. As for the method of numerical analysis, many worker have already been reported[1][4]. But a number of numerical methods have used Green's function approach with TEM-mode approximation and, hence, are not the fact which paid attention to the two-dimensional EM field distribution. However, since a H-plane short-boundary planar circuit is a perfectly closed system, the direct measurement of the EM field distribution in the planar circuit is very difficult. To obtain the EM field distribution in this circuit, a special technique is required. In this paper, we are proposed a method for calculating and measuring the two-dimensonal EM field distribution in the planar circuits, making use of a kind of analogy existing between a H-plane short-boundary planar circuit and a trough-type surface-wave planar circuit.

\section{Electromagnetic Field Distribution \\ A. Geometry and Basic Equations}

Before starting the derivation of the basic relations,let us briefly review the planar circuit structure treated here. We consider a structure of (a) H-plane waveguidetype planar circuit and (b) trough-type surface-wave

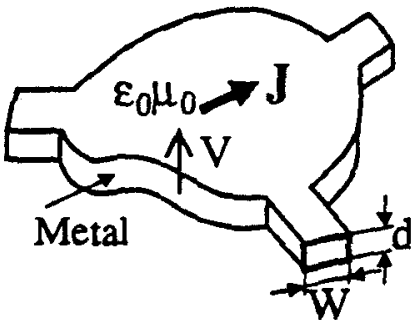

(a) H-plane short boundary planar circuit

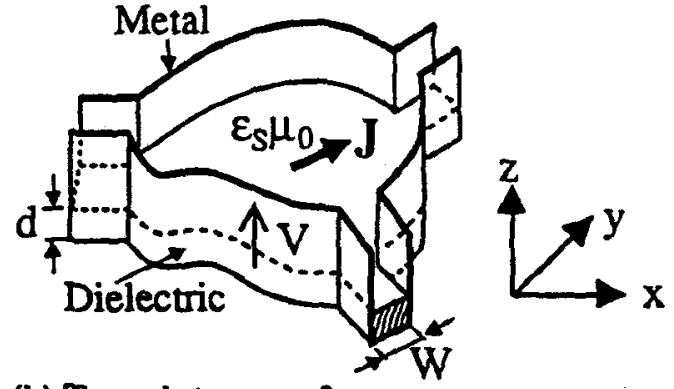

(b) Trough-type surface-wave planar circuit

Fig.1 Structure of planar circuit

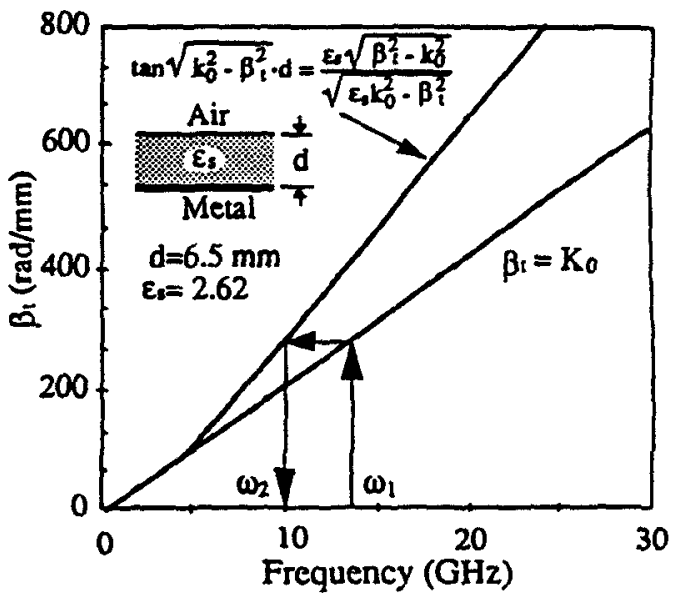

Fig 2 Dispersion curve of dielectric slab

planar circuit having the same metal-wall boundary conditions as shown in Fig.1(a),(b). Then, the field component in the planar circuit is as follows.

$$
\mathbf{E}=\left(\mathbf{E}_{\mathbf{1}}, \mathrm{E}_{\mathrm{z}}\right), \mathrm{H}=\left(\mathrm{H}_{\mathrm{x}}, \mathrm{H}_{\mathbf{y}}, \mathbf{0}\right)
$$

Voltage and currents densities in the planar circuit are defined as $V(x, y)=-E_{2} d, J(x, y)=\left(H_{y}, H_{x}\right)$ 


\begin{tabular}{|c|c|c|c|}
\hline $\begin{aligned} \operatorname{grad} V(x, y) & =-j \frac{k_{0}^{2} d}{\omega \varepsilon_{0}} J(x, y) \\
\operatorname{div} J(x, y) & =-j \frac{\omega \varepsilon_{0}}{d} V(x, y)\end{aligned}$ & (2) & $\begin{aligned} \operatorname{grad} V(x, y) & =-j \frac{\beta_{1}^{2} d}{\omega \varepsilon_{0}} J(x, y) \\
\operatorname{div} J(x, y) & =-j \frac{\omega \varepsilon_{0}}{d} V(x, y)\end{aligned}$ & $(2)^{\prime}$ \\
\hline $\mathrm{k}_{0}^{2}=\omega \sqrt{\varepsilon \mu}$ & (3) & $\tan \sqrt{\mathrm{k}_{0}^{2}-\beta_{\mathrm{t}}^{2}} \mathrm{~d}=\frac{\varepsilon_{\mathrm{s}} \sqrt{\beta_{\mathrm{t}}-\mathrm{k}_{0}^{2}}}{\sqrt{\varepsilon_{\mathrm{s}} \mathrm{k}_{0}^{2}-\beta_{\mathrm{t}}^{2}}}$ & $(3)^{\prime}$ \\
\hline$\nabla_{\mathrm{t}}^{2} \mathrm{~V}+\mathrm{k}_{0}^{2} \mathrm{~V}=0$ & (4) & $\nabla_{\mathrm{t}}^{2} \mathrm{~V}+\beta_{\mathrm{t}}^{2} \mathrm{~V}=0$ & $(4)^{\prime}$ \\
\hline
\end{tabular}

The Basic planar cincuit equations (2),(3),(4) in both planar circuits and Helmholtz equation with regard to voltage are derived from Maxwell's equations respectively[2],[3]. In these relations, both planar circuits have essentially the same planar circuit equations and boundary conditions except the lateral wavenumber $\mathrm{k}_{0}$ and $\beta_{\mathrm{t}}$. Therefore, Referring to an $\omega-\beta$ diagram shown in Fig.2, if $k_{0}\left(\omega_{1}\right)=\beta_{1}\left(\omega_{2}\right)$ in both planar circuits, the input/output frequency characteristics and the EM field distributions become the same property.

Using the relation of this analogy, and the frequency translation, the property of frequency $\omega_{1}$ in (a) is correspond to the ones of frequency $\omega_{2}$ in $(b)$ and vice versa. That is, the former is difficult to measure the EM field due to closed-system structure, but the latter can be easily measured the EM field on uniform dielectric sheet due to upper-open structure.

\section{B. Electric Field Distribution in Planar Circuit}

When the planar circuit is excited by TE10 mode incidence at the input/output coupling waveguide, the EM field distribution is described using Green' function $\mathbf{G}\left(\mathbf{r} ; \mathbf{r}_{0}\right)$ at $(x, y, d)$ due to a unit current density source located at the source point $\mathbf{r}_{0}$

$$
\nabla_{\mathfrak{t}}^{2} \mathbf{G}+\mathrm{k}^{2} \mathbf{G}=-\delta\left(\mathbf{r}-\mathrm{r}_{0}\right)
$$

where $\mathbf{G}\left(\mathbf{r}, \mathbf{r}_{0}\right)$ satisfies the following boundary conditions $\quad \mathbf{G}=0$ on $C$ (periphery)

$\partial \mathbf{G} / \partial \mathbf{n}=0$ on $\mathrm{Cw} \quad$ (coupling port parts)

For a surface current distribution $\mathbf{J}_{\mathbf{S}}$ on the coupling port connected in the planar circuit, two-dimensional normal component $V(x, y)$ is given by

$$
\mathrm{V}(\mathrm{x}, \mathrm{y})=\mathrm{Z} \int \mathbf{G}\left(\mathbf{r} ; \mathbf{r}_{0}\right) \mathrm{J}_{\mathrm{s}} \mathrm{dxdy}
$$

$$
\text { where } Z=j k_{0}^{2} d / \omega \varepsilon_{0} \text {, or } j \beta_{t}^{2} d / \omega \varepsilon_{0}
$$

Since the $\mathbf{G}\left(\mathbf{r}, \mathbf{r}_{0}\right)$ can be expressed in terms of the set of eigenfunctions having the same boundary conditions, then, $E z(x, y)$ can be calculated by

$$
\begin{aligned}
& E_{z}(x, y)= \sum_{n=1}^{\infty}\left[\frac{j \omega \mu}{\omega^{2} \varepsilon \mu-k_{n}^{2}} \frac{1}{S}\right. \\
&\left.\cdot \sum_{j=1}^{m} \sum_{q=1}^{\infty} n_{q n}^{(j)} I_{q}^{(j)}\right] \cdot \varphi_{n}(x, y)
\end{aligned}
$$

where $n_{n, q}^{(j)}$ is ideal transformer ratio between $n$-th planar mode and q-th mode of $j$-th transmission line and given by eq. (8).

$$
\begin{aligned}
& n_{q n}^{(j)}=\frac{1}{w^{(j)}} \int_{0}^{(j)} \varphi_{n}\left(x_{0}, y_{0}\right) \cdot f_{q}^{(j)}\left(s^{(j)}\right) d s^{(j)} \\
& J_{q}^{(j)}\left(s^{(j)}\right)=I_{q}^{(j)} / W(j) \int_{0}^{w(j)} f_{q}^{(j)}\left(s^{(j)}\right) d s^{(j)} \\
& f_{q}^{(j)}\left(s^{(j)}\right)=\sqrt{2} \sin \frac{q \pi s^{(j)}}{w^{(j)}}
\end{aligned}
$$

(eigenfunction of transmission-line)

\section{Experimental Method and Measurement Setup}

Fig.3 shows a block-diagram for measuring the twodimensional EM field distribution in the trough-type planar circuit. As described above, since the field component $E_{z}(x, y)$ in trough-type planar circuit is always normal to dielectric sheet, we set a probe antenna upright on dielectric sheet and the measurement proceeds by sliding the electric probe over the $x-y$ surface of dielectric sheet,holding it at a constant height position. Using the measurement equipment shown in Fig.3. the two-dimensional electric field distribution is detected. Practically, the probe-antenna is mounted on a movable rack mechanism that maintains the distance between the probe tips and dielectric sheet to within $1 \mathrm{~mm}$ as the probe slides over the $x-y$ surface, using the computer controlled stepping motor and sampling the EM field through 12 bit A/D convertor, and are stored in memory $(x$-direction maximum movable-length $100 \mathrm{~mm}$, $y$-direction maximum movable-length $130 \mathrm{~mm}$ ).

Furthermore, the electric probe is formed by mounting on semi-rigid cable such that only the $2 \mathrm{~mm}$ long center conductor protruded into the enclosure.

\section{Numerical and Experimental Results}

In order to confirm the validity and usefulness of the field measurement system based upon the analogy existing between both planar circuits, we show calculated and measured some typical examples of the frequency response of two-dimensional electric field distribution for $\mathrm{H}$-plane short-boundary planar circuits.

To calculate the frequency response of the electric field distribution by the planar circuit approach, we take into account 30 eigenmodes in the planar circuit and 4 higher-order modes in the transmission-line. 
A. Frequency response of $E M$ field distribution of right angle corner-cut bend

Fig.4 shows calculated frequency characteristics of $\left|S_{i j}\right|^{p}$ with parameters comer-cut $\mathrm{C}=\mathrm{C} / \mathrm{W}$, and two dimensional electric field distributions for $\mathrm{H}$-plane waveguide right angle comer-cut bend. From these results, it can be seen that the proper corner-cut plays an important role in smoothing the field distribution, which results in a reduction of the reflections at the discontinuity.

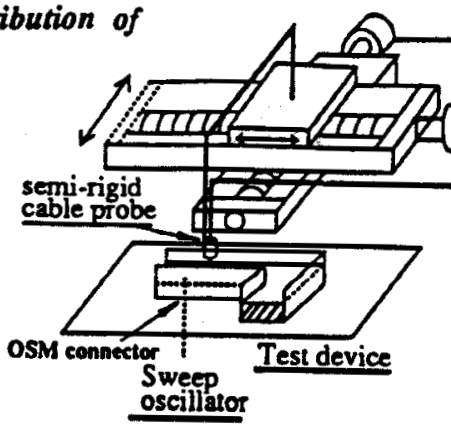

Fig.3 Block diagram of two-dimensional electric

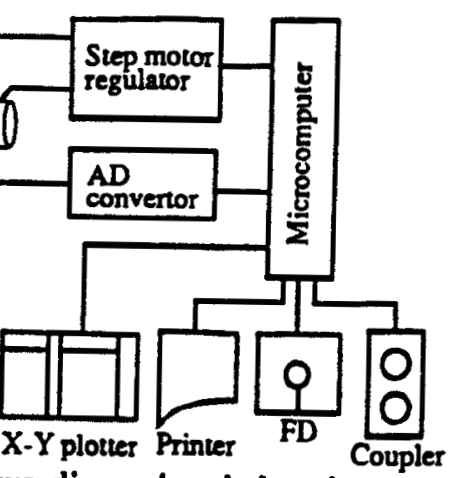
field measurement setup
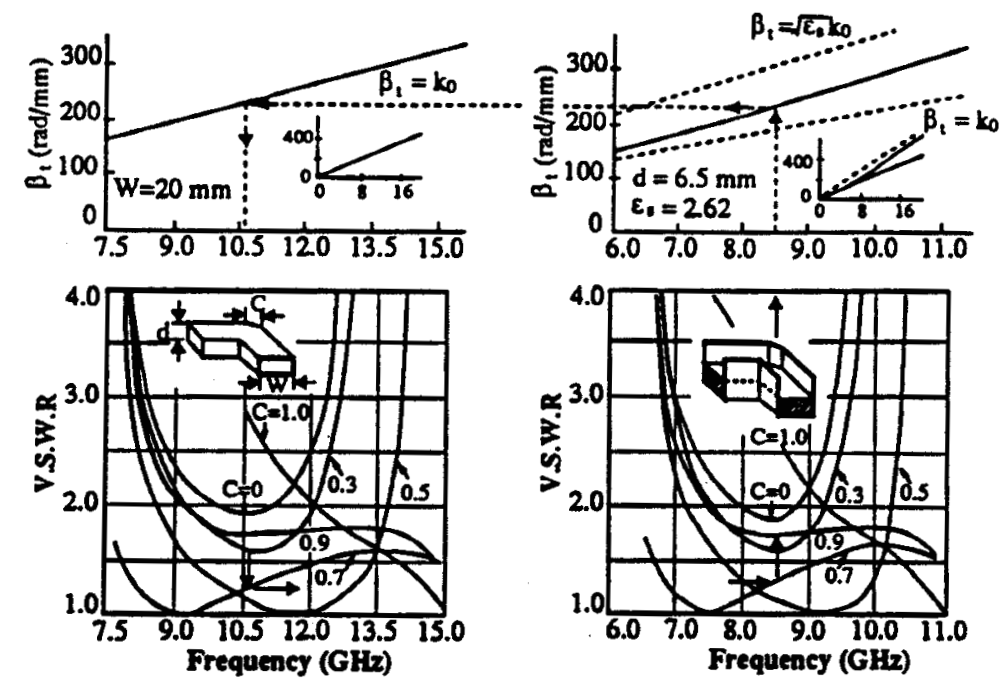

(a) Frequency characteristics
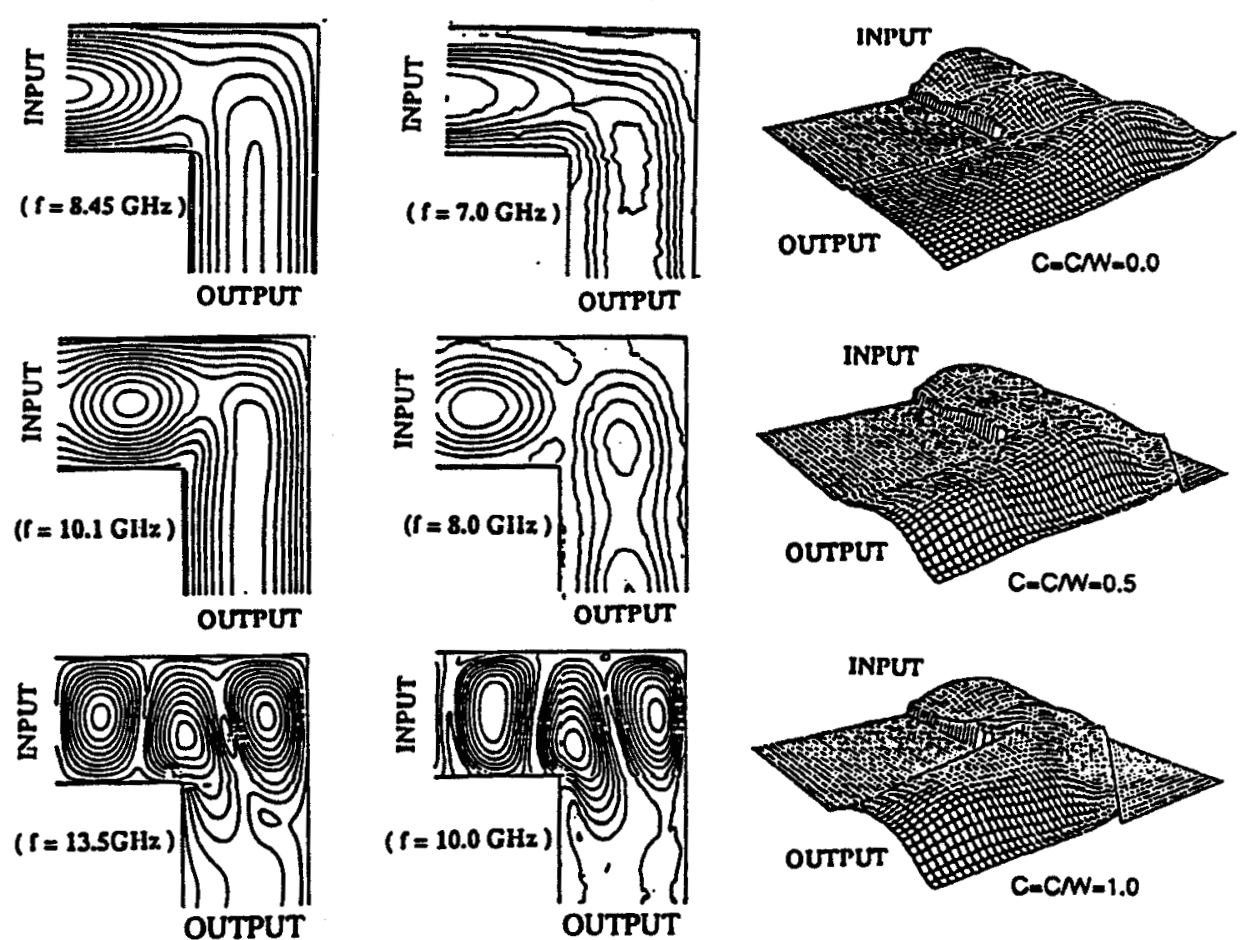

(b) Calculated field

(c) Measured field

(d) Measured field $(f=9.0 \mathrm{GHz}$ )

Fig.4 Frequency characteristics and electric field distribution of H-plane waveguide right angle corner bend.

865. 


\section{B. Electric field distribution of $H$-plane waveguide T-bifurcation}

Fig.5 shows calculated frequency characteristics of $\left|S_{i}\right|^{2}$ as a function of the frequency and the electric field distributions for $\mathrm{H}$-plane waveguide $\mathrm{T}$-bifurcation.

\section{Conclusion}

A method for calculating and measuring the twodimensional EM field distribution in both planar circuits is proposed, making use of the analogy between the $\mathrm{H}$ plane short-boundary planar circuit and the trough-type surface wave planar circuit having the same boundary conditions. The agreement between theory and measurement is quite satisfactory. Thus, the proposed measurement system offers a valid means of predicting the theoretical analysis of more complicated discontinuity problems. The method of the electric field distribution measurement described here will be also applied to various $\mathrm{H}$-plane planar structures.
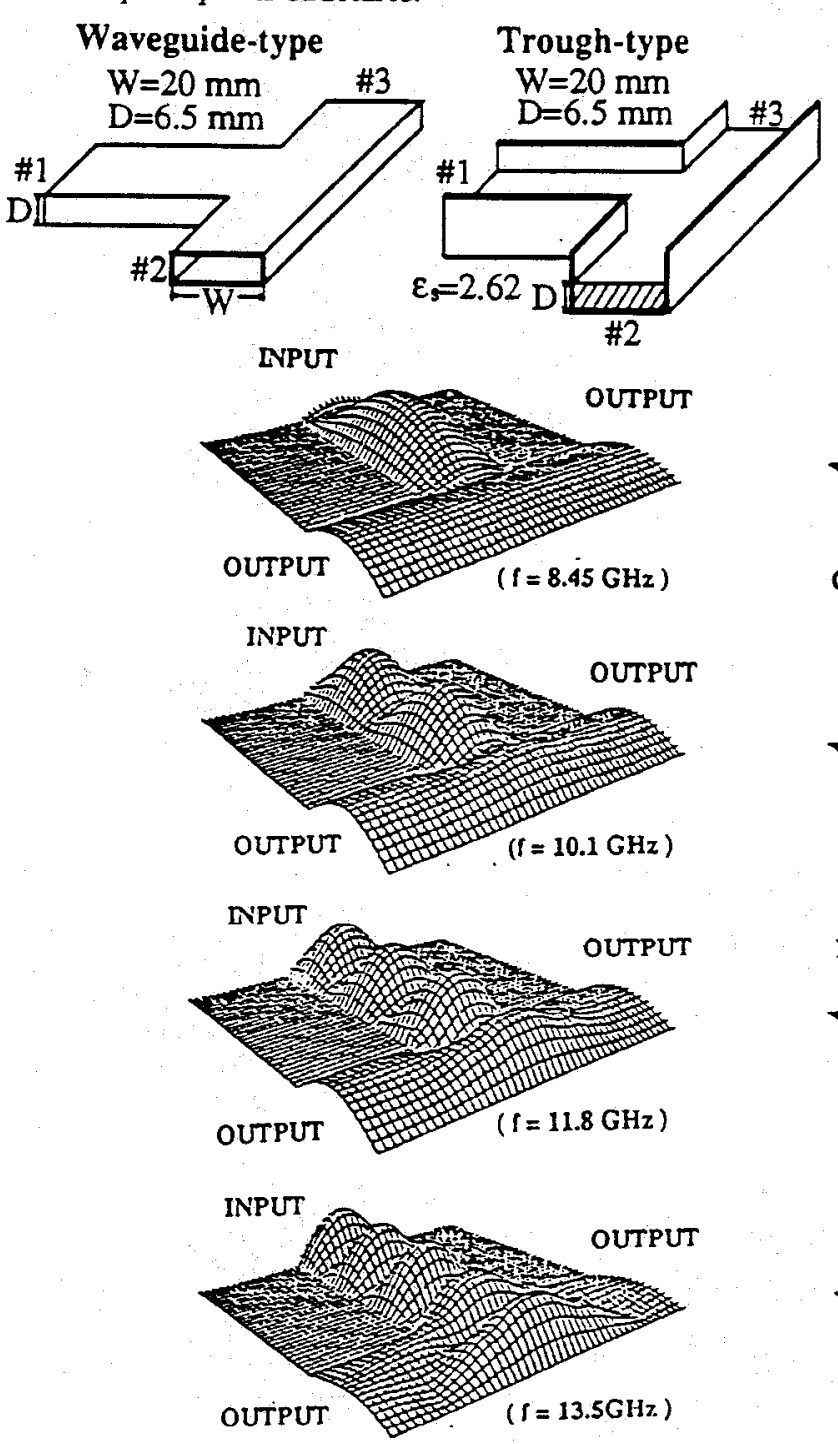

(b) Calculated field

\section{Reference}

(1)T.Okoshi and T.Miyoshi, "The Planar Circuit-An Approach to Microwave integrated Circuitry", IEEE MTT.vol.20.,No.4 pp.245-252, April 1972.

(2)Hsu, jui-Pang and T. Anada, "Planar Circuit Equation and Its Practical Application to Planar-type transmission Line Circuit",MTT Int' Symp.1983,pp.574-576 (3)Hsu, jui-Pang and T. Anada, "Proposal of surfacewave planar Circuit equations and Its Practical Application", MTT Int' Symp. GG 4,1986

(4)R. Sorrentino, "Planar circuits, waveguide models and segmentation method", IEEE Trans. MTT., vol. MTT-33, pp.1057 - 1066, Oct.1985
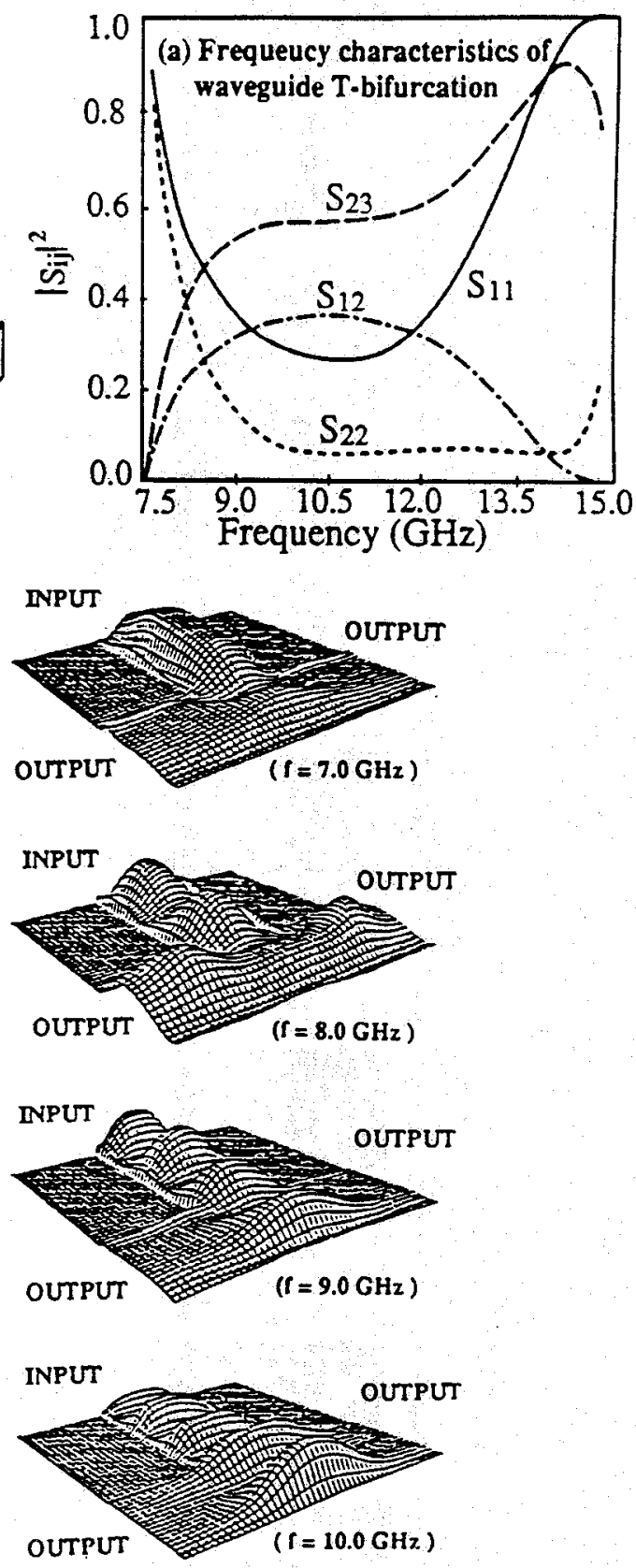

(c) Measured field

Fig. 5 Frequency characteristics and electric field distribution of $\mathrm{H}$-plane waveguide $\mathrm{T}$ - bifurcation 